\title{
Spontaneous emission and energy transfer rates near a coated metallic cylinder
}

\author{
Vasilios Karanikolas, Cristian A. Marocico, and A. Louise Bradley* \\ Semiconductor Photonics Group, School of Physics and CRANN, Trinity College Dublin, College Green 2, Dublin, Ireland
}

(Received 13 March 2014; published 19 June 2014)

\begin{abstract}
The spontaneous emission and energy transfer rates of quantum systems in proximity to a dielectrically coated metallic cylinder are investigated using a Green's tensor formalism. The excitation of surface plasmon modes can significantly modify these rates. The spontaneous emission and energy transfer rates are investigated as a function of the material and dimensions of the core and coating, as well as the emission wavelength of the donor. For the material of the core we consider gold and silver, whose surface plasmon wavelengths lie in the visible part of the electromagnetic spectrum. The spontaneous emission rate is enhanced by several orders of magnitude when the emission wavelength is close to the surface plasmon wavelength. The energy transfer rate enhancement is found to be concentrated in hot spots around the circumference of the coated cylinder. Introducing the energy transfer efficiency as a parameter, we find that, when the donor emission and acceptor absorption spectra are resonant with the surface plasmon modes excited on the coated cylinder, the energy transfer efficiency can be significantly enhanced compared to the off-resonance situation. Tuning the surface plasmon wavelength to the emission wavelength of the donor via the geometrical and material parameters of the coated cylinder allows, therefore, control of the energy transfer efficiency.
\end{abstract}

DOI: 10.1103/PhysRevA.89.063817

PACS number(s): 33.80.-b, 42.50.-p, 73.20.Mf

\section{INTRODUCTION}

In 1946 Purcell showed that a quantum system's intrinsic properties, such as its spontaneous emission (SE) or decay rate, can be modified by the presence of material bodies in its vicinity [1]. Various structures have been investigated with respect to their role in modifying the SE rate: planar dielectric and metallic interfaces [2-8], spheres [9-13], and dielectric and metallic cylinders [14-22].

The energy transfer (ET) rate between two quantum systems, donor and acceptor, can also be influenced by the environment. A variety of geometrical arrangements have been considered when investigating the ET rate, e.g., planar geometries [5,8,23,24], dielectric spheres [25-28], photonic crystals [29], microcavities [30-33], etc. These investigations have found that both enhancement and reduction of the ET rate can occur.

In particular, the SE and ET rates can be dramatically enhanced when the quantum systems are placed in close proximity to metallic bodies, due to the possibility of exciting surface plasmon modes [34,35]. Surface plasmons (SPs) are collective oscillations of electrons and the electromagnetic field that can be excited at the interface between a dielectric and a conductor. Depending on the size and shape of the dielectric and conducting bodies, the SPs can either propagate along the interface or be localized. The frequency at which SP modes can be excited depends on the geometrical and material characteristics of the bodies.

The SE and ET rates have been studied extensively for cylindrical geometries during the past decade, the focus being mainly on the SE rate in the presence of dielectric and metallic cylinders. Furthermore, it has been shown that the ET rate between a pair of quantum emitters can be significantly enhanced due to coupling to SP modes on a metallic cylinder [19,22]. It is also known that the presence

\footnotetext{
*bradlel@tcd.ie
}

of a metallic coating on a dielectric core can support SP modes [18]. The presence of the dielectric coating will modify the SP dispersion relations as a function of the thickness and refractive index of the coating. This will, in turn, modify the coupling of the quantum systems to the coated cylinder and offer a way to control the SE and ET rates through the optical and geometrical parameters of the dielectric coating.

In this contribution we consider a metallic core coated with a dielectric layer and we investigate the influence of the SP modes on the SE and ET rates of quantum systems placed in proximity to the coated cylinder. These rates are calculated using a semianalytical Green's tensor method [36,37]. A variety of quantum systems can be investigated this way, e.g., atoms, molecules, quantum dots, and fluorescent dyes. The metallic core will be either $\mathrm{Ag}$ or $\mathrm{Au}$, since the SP wavelength of these metals lies in the visible part of the electromagnetic spectrum, making them of significant practical interest. Tabulated experimental data are used to describe the optical response of these materials [38].

Top-down techniques, e.g., electron-beam lithography, or bottom-up techniques, e.g., colloidal synthesis, can be used to fabricate hybrid nanostructures with dimensions of a few tens of nanometers. These structures form the building blocks for a variety of potential technological applications. Nanowires and coated nanowire structures can be used in light harvesting [39,40] and switching devices [41], imaging [42,43], light conversion $[43,44]$, cloaking $[45,46]$, and quantum optics applications [47]. A good understanding of the SE and ET processes in these environments is important for manipulation of light below the diffraction limit.

The paper is structured as follows. In Sec. II we present an outline of the formalism used, while in Sec. III the results of our simulations of the SE and ET rates are presented and discussed. Finally, Sec. IV is reserved for a summary of the results and the conclusions that can be drawn. In addition, the derivations of several expressions are given in two Appendices. 


\section{SPONTANEOUS EMISSION, ENERGY TRANSFER, AND THE GREEN'S TENSOR FORMALISM}

\section{A. Spontaneous emission rate}

Spontaneous emission is the process by which an excited quantum mechanical system spontaneously decays to its ground state. We therefore start by considering a two-level quantum system for which $|e\rangle$ and $|g\rangle$ denote the excited and ground states, respectively. The system interacts electromagnetically with its environment even in the absence of an external electromagnetic field, which leads to the spontaneous relaxation of the excited two-level system. The initial state of the system is $|i\rangle=|e\rangle \otimes|0\rangle$, where the system is in the excited state and the electromagnetic field is in its vacuum state. The two-level system will not stay excited indefinitely, but will relax to its ground state by emitting a photon and, therefore, the electromagnetic field will be in the state $|1(\mathbf{k}, \alpha)\rangle$, where $\mathbf{k}$ and $\alpha$ are the wave vector and polarization of the emitted photon, respectively. We can express this state by introducing the creation operator $\hat{f}_{i}^{\dagger}(\mathbf{r}, \omega)$ which acts on the vacuum state to generate the one-excitation state. The final state then has the form $|f\rangle=|g\rangle \otimes \hat{f}_{i}^{\dagger}(\mathbf{r}, \omega)|0\rangle$. The creation and annihilation operators $\hat{f}_{i}^{\dagger}(\mathbf{r}, \omega)$ and $\hat{f}_{i}(\mathbf{r}, \omega)$, respectively, can also account for medium dressed states such as SPs which are a main focus of this paper [48].

Applying Fermi's golden rule and summing over all final states, we obtain an expression for the SE rate, $\gamma[13,49]$,

$$
\gamma(\mathbf{r}, \omega)=\frac{2 \omega^{2} \mu^{2}}{\hbar \varepsilon_{0} c^{2}} \operatorname{Im}\left[\mathbf{n}_{\mu} \cdot \mathfrak{G}(\mathbf{r}, \mathbf{r}, \omega) \cdot \mathbf{n}_{\mu}\right],
$$

where $\mathbf{n}_{\mu}$ is a unit vector along the direction of the transition dipole moment of the emitter, $\mu$, and $\mathfrak{G}(\mathbf{r}, \mathbf{r}, \omega)$ is the Green's tensor representing the response of the geometry under consideration to a pointlike excitation, located at $\mathbf{r}$. The Green's tensor is a solution of the differential equation

$$
\nabla \times \nabla \times \mathfrak{G}(\mathbf{r}, \mathbf{s}, \omega)-\frac{\omega^{2}}{c^{2}} \varepsilon(\mathbf{r}, \omega) \mathfrak{G}(\mathbf{r}, \mathbf{s}, \omega)=\mathfrak{I} \delta(\mathbf{r}-\mathbf{s}),
$$

where $\mathfrak{I}$ is the unit $3 \times 3$ tensor and $\varepsilon(\omega)$ represents the spatially dependent complex permittivity of the system. The $i j$ component of the Green's tensor represents the $j$ component of the electric field at position $\mathbf{r}$ created by a point dipole source oriented along direction $i$ and located at position $\mathbf{s}$.

A useful quantity to introduce is the normalized SE rate, defined as

$$
\tilde{\gamma}=\frac{\gamma}{\gamma_{0}}=n_{i}+\frac{6 \pi c}{\omega} \operatorname{Im}\left[\mathbf{n}_{\mu} \cdot \mathfrak{G}_{s}(\mathbf{r}, \mathbf{r}, \omega) \cdot \mathbf{n}_{\mu}\right],
$$

where the expression for $\gamma_{0}$ is given by the Einstein $A$ coefficient as $\gamma_{0}=\omega^{3} \mu^{2} /\left(3 \pi \hbar \varepsilon_{0} c^{3}\right)$. The subscript $s$ on the Green's tensor denotes the scattering part of this quantity, which we introduce in Eqs. (8), Sec. II C. The final equality in Eq. (3) above was obtained from Eq. (1), and $n_{i}=\sqrt{\varepsilon_{i}}$ is the refractive index of the medium into which the quantum system is embedded. The normalized SE rate from Eq. (3) gives either an enhancement $(\tilde{\gamma}>1)$ or a reduction $(\tilde{\gamma}<1)$ of the SE rate compared to its free-space value, $\gamma_{0}$.

When dealing with statistical ensembles of emitters, the emission spectrum will be different from that of a single emitter, which we have taken to have a $\delta$ shape. The emission rate for the ensemble can then be expressed as

$$
k_{\mathrm{SE}}=\int_{0}^{\infty} d \lambda f_{\mathrm{D}}(\lambda) \gamma(\lambda)
$$

where $f_{\mathrm{D}}(\lambda)$ is the area-normalized emission spectrum of the emitter, with $\int_{0}^{\infty} d \lambda f_{\mathrm{D}}(\lambda)=1$.

\section{B. Energy transfer rate}

The process of ET considers the transfer of the excitation energy of an excited donor quantum system to an acceptor quantum system that is in its ground state. We can, therefore, consider a pair of two-level quantum systems, D and A, and study the ET between them. The initial state is $|i\rangle=$ $\left|e_{\mathrm{D}}\right\rangle \otimes\left|g_{\mathrm{A}}\right\rangle \otimes|0\rangle$, where $\left|e_{\mathrm{D}(\mathrm{A})}\right\rangle$ and $\left|g_{\mathrm{D}(\mathrm{A})}\right\rangle$ are the excited and ground states of the donor D (acceptor A), while $|0\rangle$ is the vacuum state of the electromagnetic field. In the final state $|f\rangle$, the donor D has relaxed to the ground state, transferring its excitation energy to the acceptor $A$, now in the excited state; the electromagnetic field is still in the vacuum state $|f\rangle=\left|g_{\mathrm{D}}\right\rangle \otimes\left|e_{\mathrm{A}}\right\rangle \otimes|0\rangle[50]$.

We find that the ET rate, $\Gamma$, between a donor-acceptor pair has the form [48]

$$
\Gamma(\omega)=\frac{2 \pi}{\hbar^{2}}\left(\frac{\omega_{0}^{2}}{c^{2} \varepsilon_{0}}\right)^{2}\left|\boldsymbol{\mu}_{\mathrm{A}} \cdot \mathfrak{G}\left(\mathbf{r}_{\mathrm{A}}, \mathbf{r}_{\mathrm{D}}, \omega\right) \cdot \boldsymbol{\mu}_{\mathrm{D}}\right|^{2}
$$

where again $\mathfrak{G}\left(\mathbf{r}_{\mathrm{A}}, \mathbf{r}_{\mathrm{D}}, \omega\right)$ is the Green's tensor for the particular geometry, $\mathbf{r}_{\mathrm{D}(\mathrm{A})}$ is the position of the donor $\mathrm{D}$ (acceptor $\mathrm{A}$ ), and $\boldsymbol{\mu}_{\mathrm{D}(\mathrm{A})}$ is the transition dipole moment of the donor $\mathrm{D}$ (acceptor A). The above expression for the ET rate depends on the donor-acceptor pair through the emission frequency of the donor and the transition dipole moment of the donor and acceptor. The influence of the geometry is completely encapsulated in the Green's tensor, being proportional to the electric field intensity, through the square of the Green's tensor.

To consider only the influence of the geometry on a general donor-acceptor pair, we now introduce the normalized ET function for the coated cylinder, $\tilde{\Gamma}$, defined as

$$
\tilde{\Gamma}(\omega)=\frac{\Gamma(\omega)}{\Gamma_{0}(\omega)}=\frac{\left|\mathbf{n}_{\mathrm{A}} \cdot \mathfrak{G}\left(\mathbf{r}_{\mathrm{A}}, \mathbf{r}_{\mathrm{D}}, \omega\right) \cdot \mathbf{n}_{\mathrm{D}}\right|^{2}}{\left|\mathbf{n}_{\mathrm{A}} \cdot \mathfrak{G}_{0}\left(\mathbf{r}_{\mathrm{A}}, \mathbf{r}_{\mathrm{D}}, \omega\right) \cdot \mathbf{n}_{\mathrm{D}}\right|^{2}},
$$

where $\mathfrak{G}_{0}\left(\mathbf{r}_{\mathrm{A}}, \mathbf{r}_{\mathrm{D}}, \omega\right)$ is the Green's tensor in free space and $\mathbf{n}_{\mathrm{D}(\mathrm{A})}$ is a unit vector in the direction of $\boldsymbol{\mu}_{\mathrm{D}(\mathrm{A})}$. As the reader can readily recognize, this is, in essence, the ratio of the intensities of the electric field created by the donor dipole in the presence and absence of the coated cylinder.

Analogously to the case of the SE rate, when considering statistical ensembles of donors and acceptors, the donor emission spectrum $f_{\mathrm{D}}(\lambda)$ and acceptor absorption cross section $\sigma_{\mathrm{A}}(\lambda)$ need to be taken into account when calculating the ET rate. We, therefore, have [51]

$$
k_{\mathrm{ET}}=\frac{36 \pi^{2} Y_{D}}{k_{\mathrm{SE}}} \int_{0}^{\infty} \frac{d \lambda}{\lambda^{2}} f_{\mathrm{D}}(\lambda)\left|\mathbf{n}_{\mathrm{A}} \cdot \mathfrak{G}\left(\mathbf{r}_{\mathrm{A}}, \mathbf{r}_{\mathrm{D}}, \omega\right) \cdot \mathbf{n}_{\mathrm{D}}\right|^{2} \sigma_{\mathrm{A}}(\lambda) .
$$

We use this expression to calculate the ET rate between donors and acceptors with specific emission and absorption spectra 


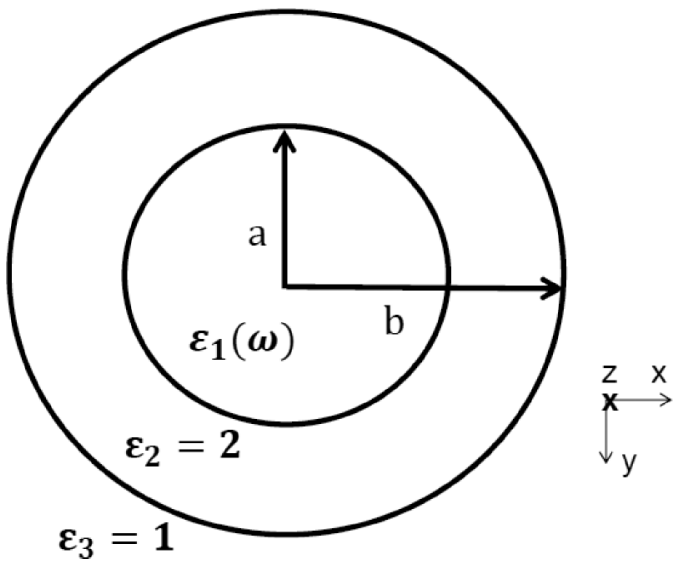

FIG. 1. Cross section of an infinitely long coated cylinder geometry, with its axis along the $z$ direction, core radius $a$, and outer radius $b$. The dielectric permittivity of the core is $\varepsilon_{1}$, the dielectric permittivity of the coating is $\varepsilon_{2}$, and that of the surrounding medium is $\varepsilon_{3}$.

and investigate how the ET process competes with the emission process of the donor.

\section{The Green's tensor of a coated cylinder}

The Green's tensor encapsulates all the information for the response of material bodies to electromagnetic fields and determines the normalized SE rate, Eq. (3), and normalized ET function, Eq. (6). In what follows we present a method for calculating the Green's tensor for the coated cylinder geometry [52].

We consider a coated cylinder with core radius, $a$, and outer radius, $b$, embedded in a homogeneous medium with dielectric permittivity $\varepsilon_{3}$, as in Fig. 1 . The cylinder axis is along the $z$ direction. The dielectric permittivity for the core cylinder is $\varepsilon_{1}$ which will be given by tabulated experimental data [38], while the coating will have a constant dielectric permittivity $\varepsilon_{2}$. Due to the cylindrical symmetry of the structure, throughout this paper we use the cylindrical coordinate system, $(\rho, \theta, z)$.

In order to calculate the Green's tensor for the coated cylinder geometry we use the method of scattering superposition [36,37]. When the source point is located in the background medium outside the coated cylinder, the Green's tensor has the form

$$
\begin{aligned}
& \mathfrak{G}^{(13)}(\mathbf{r}, \mathbf{s}, \omega)=\mathfrak{G}_{s}^{(13)}(\mathbf{r}, \mathbf{s}, \omega), \\
& \mathfrak{G}^{(23)}(\mathbf{r}, \mathbf{s}, \omega)=\mathfrak{G}_{s}^{(23)}(\mathbf{r}, \mathbf{s}, \omega), \\
& \mathfrak{G}^{(33)}(\mathbf{r}, \mathbf{s}, \omega)=\mathfrak{G}_{h}^{(33)}(\mathbf{r}, \mathbf{s}, \omega)+\mathfrak{G}_{s}^{(33)}(\mathbf{r}, \mathbf{s}, \omega),
\end{aligned}
$$

where the first of the two labels in the superscript (i3) denotes the field point, while the second denotes the source point. The subscript $s$ denotes the scattering term, always present, while the homogeneous term $\mathfrak{G}_{h}^{(33)}(\mathbf{r}, \mathbf{s}, \omega)$ contributes only when the source and field points are in the same medium. When calculating the ET rate, the source and field points correspond to the donor and acceptor positions, respectively, while for the $\mathrm{SE}$ rate, the source and field points coincide and correspond to the position of the quantum emitter.
The scattering term has the following general expression

$$
\begin{aligned}
\mathfrak{G}_{s}^{(i 3)}(\mathbf{r}, \mathbf{s}, \omega)= & \frac{i}{8 \pi} \sum_{n, K} \int_{-\infty}^{\infty} d k_{z}\left[R_{M K}^{i 3} \mathbf{M}_{n}\left(k_{\rho i}, \mathbf{r}\right)\right. \\
& \left.+R_{N K}^{i 3} \mathbf{N}_{n}\left(k_{\rho i}, \mathbf{r}\right)\right] \otimes \mathbf{K}_{n}^{(1)}\left(k_{\rho 3}, \mathbf{s}\right),
\end{aligned}
$$

where $k_{\rho i}=\sqrt{k_{i}^{2}-k_{z}^{2}}$ is the radial propagation constant in medium $i$, and $k_{i}=\frac{\omega}{c} \sqrt{\varepsilon_{i}}$ is the wave number in medium $i$ $(i=1,2,3)$. The above expression involves a summation over $\mathbf{K}$, which represents $\mathbf{M}_{n}^{(1)}\left(k_{\rho 3}\right)$ and $\mathbf{N}_{n}^{(1)}\left(k_{\rho 3}\right)$, or the transverse electric (TE) and transverse magnetic (TM) modes. As is evident, the field has a hybrid nature and cannot be separated into TE and TM modes. The vector wave functions are solutions of the Helmholtz equation in cylindrical coordinates and involve Bessel and Hankel functions. The superscript (1) in $\mathbf{M}_{n}^{(1)}\left(k_{\rho}\right)$ denotes the fact that we use the Hankel function of the first kind. This form of the Green's tensor already takes into account the radiation condition at infinity and the regularity condition on the $z$ axis, i.e., at $\rho=0$, where the regular Bessel functions are used.

We impose the following continuity conditions at the surface of the core $\left(\rho_{1}=a\right)$ and coating $\left(\rho_{2}=b\right)$,

$$
\begin{array}{r}
\hat{\rho} \times\left.\left[\mathfrak{G}^{(i 3)}(\mathbf{r}, \mathbf{s}, \omega)-\mathfrak{G}^{[(i+1) 3]}(\mathbf{r}, \mathbf{s}, \omega)\right]\right|_{\rho=\rho_{i}}=0, \\
\hat{\rho} \times\left.\left[\nabla \times \mathfrak{G}^{(i 3)}(\mathbf{r}, \mathbf{s}, \omega)-\nabla \times \mathfrak{G}^{[(i+1) 3]}(\mathbf{r}, \mathbf{s}, \omega)\right]\right|_{\rho=\rho_{i}}=0 .
\end{array}
$$

Using Eqs. (8) in the continuity conditions we obtain two inhomogeneous systems of linear equations, one for each polarization $\mathbf{M}$ or $\mathbf{N}$. We can write this system of equations in matrix form as

$$
\Delta\left(n, k_{z}\right) \cdot \mathbf{R}_{M(N)}\left(n, k_{z}\right)=\mathbf{V}_{M(N)}\left(n, k_{z}\right),
$$

where $\Delta\left(n, k_{z}\right)$ represents the characteristic matrix, the same for both polarizations TE and TM, $\mathbf{R}_{M}\left(n, k_{z}\right)$ and $\mathbf{R}_{N}\left(n, k_{z}\right)$ are the unknown coefficients vectors, and $\mathbf{V}_{M}\left(n, k_{z}\right)$ and $\mathbf{V}_{N}\left(n, k_{z}\right)$ are the free-term vectors, known quantities emerging from the homogeneous part of the Green's tensor $\mathfrak{G}^{(33)}(\mathbf{r}, \mathbf{s}, \omega)$, and associated with the field of a point dipole source. The expressions for the characteristic matrix and the coefficients and free-term vectors are given in Appendix A.

As an example, we give the expression of the scattering part of the Green's tensor for the case when the donor and the acceptor are both oriented along the $z$ direction:

$$
\begin{aligned}
\mathfrak{G}_{s(z z)}^{(13)}(\mathbf{r}, \mathbf{s}, \omega)= & \frac{i}{8 \pi} \sum_{n=-\infty}^{\infty} \int_{-\infty}^{\infty} d k_{z} \frac{k_{\rho 1}^{2}}{k_{1} k_{3}} R_{N N}^{13} J_{n}\left(k_{\rho 1} \rho\right) \\
& \times H_{n}\left(k_{\rho 3} \rho_{s}\right) e^{i n \theta} e^{i k_{z} z}
\end{aligned}
$$

The integrals above do not depend on $\theta_{s}$, because of the rotational symmetry, or $z_{s}$, because of translation symmetry along the axis of the cylinder. The integrals are also symmetric with respect to $k_{z}$, which means that they can be replaced with their symmetrized versions, with $k_{z}$ in the interval $(0, \infty)$. The various scattering coefficients have a complicated form, and we implement numerical methods to calculate these integrals and thereafter the SE and ET rates. One can distinguish three contributions to the above integrals: for $k_{z} \in\left(0, k_{3}\right)$, one 


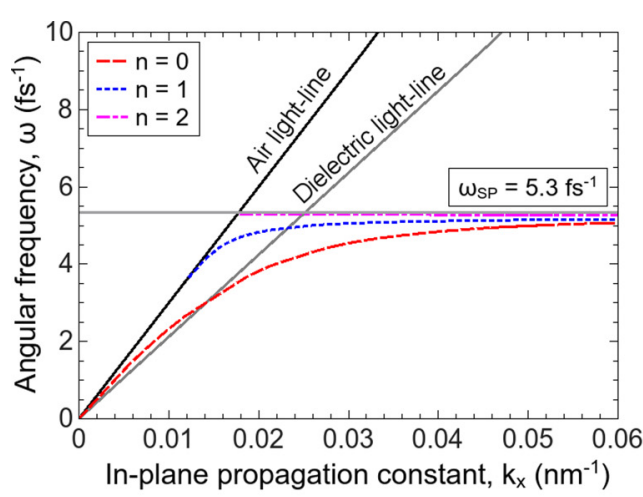

(a)

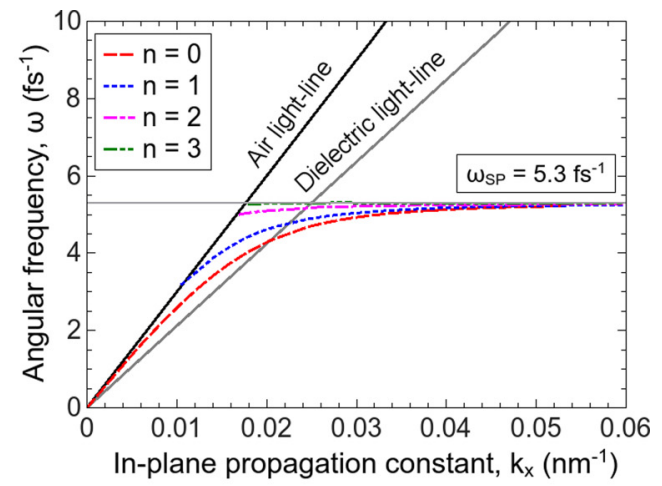

(b)

FIG. 2. (Color online) Dispersion curves of SPs of different orders $n$ on a coated cylinder with a Ag core, dielectric coating $\varepsilon_{2}=2$ and embedded in air, for two different sets of geometric parameters: (a) $a=40 \mathrm{~nm}$ and $b=50 \mathrm{~nm}$; (b) $a=80 \mathrm{~nm}$ and $b=90 \mathrm{~nm}$.

integrates over contributions from radiative modes traveling freely in the surrounding medium, i.e., air; for $k_{z} \in\left(k_{3}, k_{2}\right)$, the integral gives the contribution from guided modes within the dielectric coating. These modes are evanescent in the surrounding medium and thus only contribute when the donor and acceptor are both close to the surface of the coating. Finally, for $k_{z} \in\left(k_{2}, \infty\right)$, the integral has contributions from SP modes on the metallic core [20], as well as from lossy surface waves with larger values of $k_{z}$ [17].

If we wish to obtain the normal modes of the geometry under consideration, we have to set the free vectors to zero in Eq. (11) and solve the resulting homogeneous system of equations [18]. This kind of system has a nontrivial solution only when the determinant of the characteristic matrix $\Delta\left(n, k_{z}\right)$ is set to zero. Due to the complex form of the $\Delta\left(n, k_{z}\right)$ we solve this equation numerically. We consider the case of a Ag core, where experimental data are used for the dielectric permittivity, $\varepsilon_{1}=\varepsilon_{\mathrm{Ag}}(\omega)$ [38], coated with a dielectric with $\varepsilon_{2}=2$ and embedded in air, $\varepsilon_{3}=1$. Figure 2 presents the dispersion curves for SPs of different orders $n$. In Fig. 2(a) the core radius is $a=40 \mathrm{~nm}$ and the outer radius is $b=50 \mathrm{~nm}$, while for Fig. 2(b) we have $a=80 \mathrm{~nm}$ and $b=90 \mathrm{~nm}$. The dielectric permittivity of the coating is $\varepsilon_{2}=2$ in both panels. The horizontal line with the value $\omega=\omega_{\mathrm{SP}}=5.3 \mathrm{fs}^{-1}$ represents the SP frequency, i.e., the asymptotic value of $\omega$ for $k \rightarrow \infty$, for all the SP dispersion curves. This frequency corresponds to a SP wavelength of $\lambda_{\mathrm{SP}}=355 \mathrm{~nm}$. In Fig. 2(a) the SP dispersion curves with $n>1$ lie close to the SP frequency $\omega_{\text {SP }}$, while in Fig. 2(b) the SP dispersion curves reach their asymptotic value for $n>2$. The value of $n$ above which the dispersion curves are close to $\omega_{\mathrm{SP}}$ increases with the size of the metallic core.

\section{RESULTS AND DISCUSSION}

Using the formalism developed in the previous section we now calculate the SE and ET functions and rates for quantum systems in the presence of a dielectric coated metallic cylinder, with core radius $a$ and outer radius $b$. All the results have been obtained for transition dipoles that are radially oriented, and we therefore use the $\rho \rho$ component of the Green's tensor.

\section{A. Spontaneous emission rate}

In this section we investigate the influence of the coated cylinder on the SE rate of a nearby quantum emitter. We present the normalized SE rate, $\tilde{\gamma}=\gamma / \gamma_{0}$, of the quantum emitter, which is the ratio of the SE rate in the presence of the coated metallic cylinder, $\gamma$, to the free-space SE rate, $\gamma_{0}$. Thus, the normalized SE rate, $\tilde{\gamma}$, gives the enhancement or the inhibition of the SE rate with respect to the free-space value due to the presence of the coated metallic cylinder.

We first consider the case of a coated cylinder of core radius $a=40 \mathrm{~nm}$ and outer radius $b=50 \mathrm{~nm}$. Figure 3 shows contour plots of the normalized SE rate, $\tilde{\gamma}$, as a function of the position of the emitter and its emission wavelength, for both Ag and Au cores. The normalized SE rate is enhanced close to the metallic core and for an emission wavelength close to the SP wavelength. Excitation of SPs on the coated cylinder requires momentum matching between the excitation field and the SP mode. This momentum matching is provided, in this case, by the dipole near field of the quantum emitter. Consequently, as the dipole is moved away from the metallic core, its near field cannot excite the SP modes and, at larger distances, we recover the free-space value of the SE rate. The $\mathrm{SP}$ wavelength for the $\mathrm{Ag}$ core is $\lambda_{\mathrm{Ag}}=356 \mathrm{~nm}$, while the $\mathrm{Au}$ $\mathrm{SP}$ wavelength is $\lambda_{\mathrm{Au}}=520 \mathrm{~nm}$.

In Fig. 4 we investigate the normalized SE rate as a function of the emission wavelength of the emitter, for a fixed coating thickness of $d=10 \mathrm{~nm}$, and a fixed position of the emitter, $r=a+5 \mathrm{~nm}$, in the middle of the coating. We consider four cases for the core radius, $a=10 \mathrm{~nm}, a=20 \mathrm{~nm}, a=40 \mathrm{~nm}$, and $a=90 \mathrm{~nm}$. As the core radius is increased, the SP wavelength does not change, as it does not depend on the cylinder radius; see Fig. 2. When the emission wavelength is above the SP wavelength, corresponding to frequencies below $\omega_{\text {SP }}$ in Fig. 2, the SE rate exhibits a strong dependence on the size of the core. More precisely, the SE rate is reduced as the size of the core is increased and this can be explained by the fact that in this wavelength regime, the emitter can couple to the SP modes of the coated cylinder. SPs with a larger wave number give a larger contribution to the SE rate, though their influence is more constrained to the surface of the core. For a given frequency in this range, the SP wave number varies inversely as the core size, and, therefore, smaller cores produce larger 


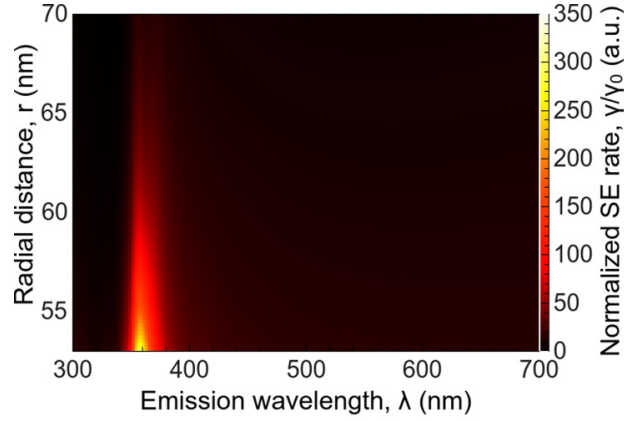

(a)

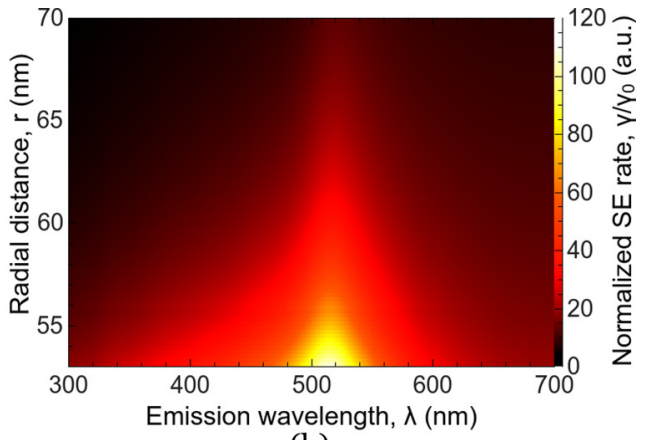

(b)

FIG. 3. (Color online) Normalized SE rate, $\tilde{\gamma}=\gamma / \gamma_{0}$, near a coated metallic cylinder as a function of the radial distance, $r$, and emission wavelength, $\lambda$, of the emitter. The core radius is $a=40 \mathrm{~nm}$ and outer radius is $b=50 \mathrm{~nm}$. The coating has constant dielectric permittivity $\varepsilon_{2}=2$ and the surrounding medium is air, $\varepsilon_{3}=1$. (a) Ag core, $\varepsilon_{1}=\varepsilon_{\mathrm{Ag}}(\omega)$; (b) Au core, $\varepsilon_{1}=\varepsilon_{\mathrm{Au}}(\omega)$. The dipole moment of the emitter is radial.

$\mathrm{SE}$ rates of the emitter. For core radii above $a=40 \mathrm{~nm}$, the SE rate no longer depends on the core size and is, in essence, the same as the SE rate for a core of infinite size, also shown in Fig. 4. When the emission wavelength is close to or below the SP wavelength, SPs of higher order and with a dispersion curve very close to the SP wavelength begin to contribute to the $S E$ rate. Coated cylinders with a larger core support a larger number of these high-order SPs which, therefore, give a larger contribution to the SE rate of the emitter. The dependence of the $\mathrm{SE}$ rate on the size of the core is then reversed, increasing with core size, though not very strongly. The limit $a \rightarrow \infty$ is seen to hold in both wavelength regimes, above and below the SP wavelength.

Apart from a larger SE rate for the Ag core, the main difference between the $\mathrm{Au}$ and the Ag cores is that the features for a $\mathrm{Ag}$ core are sharper than those for a Au core. For the latter, the dependence of the normalized SE rate, $\tilde{\gamma}$, on the emission wavelength has a broader and shallower peak around the SP wavelength. This is due to the larger absorption of $\mathrm{Au}$ compared with $\mathrm{Ag}, \operatorname{Im}\left[\varepsilon_{\mathrm{Au}}(\omega)\right]>\operatorname{Im}\left[\varepsilon_{\mathrm{Ag}}(\omega)\right]$.

In Fig. 5 we present the normalized SE rate, $\tilde{\gamma}$, as a function of the distance of the emitter from the surface of the core for the four different core radii. The emission wavelength of the quantum emitter is the same for all curves in each panel,
Fig. 5 (a) $\lambda=356 \mathrm{~nm}$ and Fig. 5 (b) $\lambda=520 \mathrm{~nm}$, respectively, placing it in the regime where the SE rate increases with the size of the core. In Fig. 5 the emitter is always outside the coating, in contrast to Fig. 4 where the emitter was placed in the middle of the coating. Because the transition dipole moment of the emitter is normal to the surface of the coating, its SE rate will have a discontinuity as the emitter moves from the coating to the background medium. As a result of this, the values of the SE rate in the background medium can be considerably different from the values inside the coating. Another consequence is that the limit $a \rightarrow \infty$ is attained for much larger values of $a \geqslant 200 \mathrm{~nm}$ when the emitter is in the background, compared to the value $a \geqslant 40 \mathrm{~nm}$ for an emitter positioned in the middle of the coating; see Fig. 4. Again, it is evident that for the Ag core, the normalized SE rate, $\tilde{\gamma}$, has larger values compared with the Au core. This different behavior of the coated cylinders with Ag and Au core can once again be attributed to the larger absorption of $\mathrm{Au}$.

The insets in the figure show the different contributions to the $\mathrm{SE}$ rate. For these calculations, we have set the losses in the metal to zero [22], to better illustrate the SP contributions. As the inset shows, the total SE rate, in the absence of losses, and the sum of the contributions from radiative and SP modes of order $n=0$ and $n=1$ overlap perfectly. When

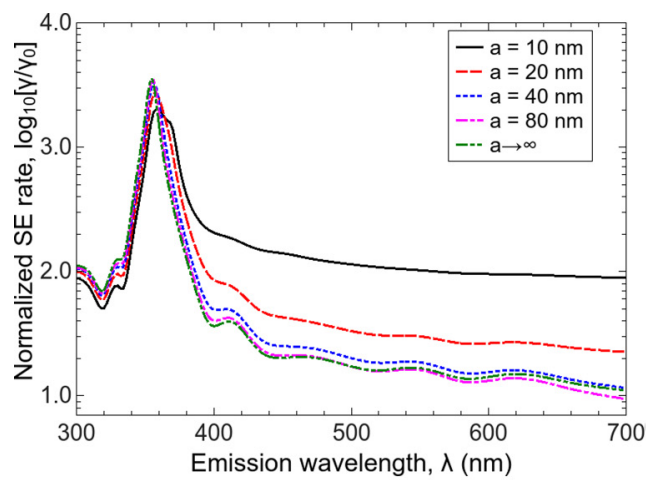

(a)

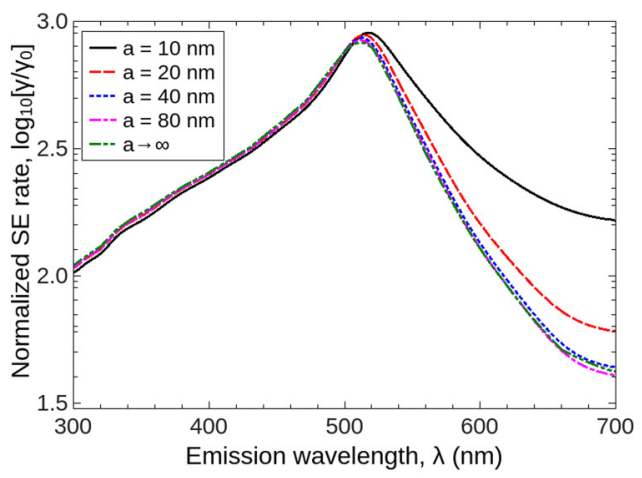

(b)

FIG. 4. (Color online) Normalized SE rate, $\tilde{\gamma}=\gamma / \gamma_{0}$, as a function of the emission wavelength, $\lambda$, of the emitter, for several core radii, $a$. The position of the emitter is fixed at $r=a+5 \mathrm{~nm}$, and its dipole moment is radial. The surrounding medium is air, $\varepsilon_{3}=1$, the coating has $\varepsilon_{2}=2$, with a fixed thickness $d=10 \mathrm{~nm}$, and the core is metallic. (a) Ag core $\varepsilon_{1}=\varepsilon_{\mathrm{Ag}}(\omega)$; (b) Au core $\varepsilon_{1}=\varepsilon_{\mathrm{Au}}(\omega)$. The SE rate in the limit $a \rightarrow \infty$ is also shown for comparison. 


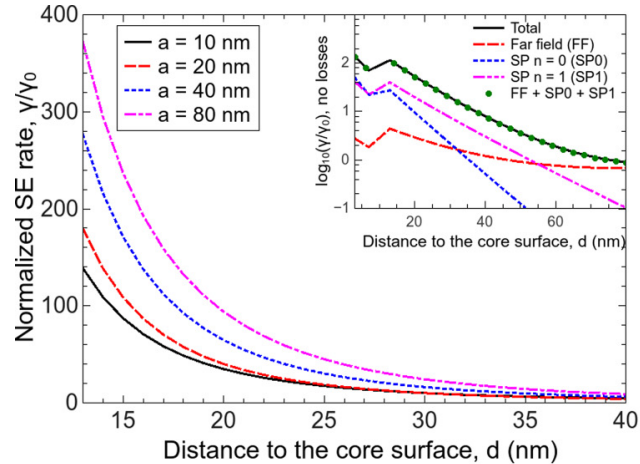

(a)

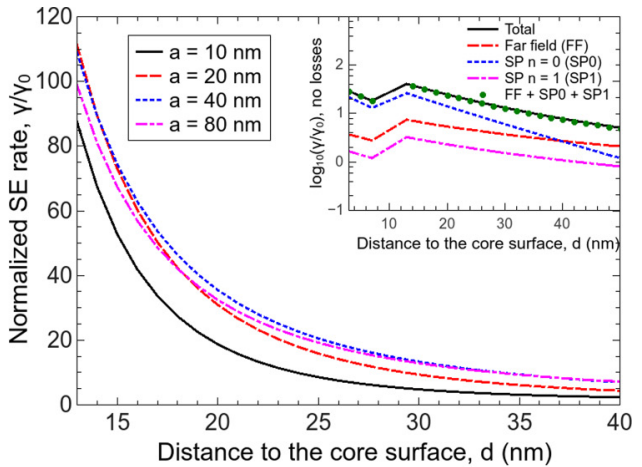

(b)

FIG. 5. (Color online) Normalized SE rate, $\tilde{\gamma}=\gamma / \gamma_{0}$, as a function of the distance of the emitter from the surface of the core, $d$, for several core radii, $a=10,20,40,80 \mathrm{~nm}$. The surrounding medium is air, $\varepsilon_{3}=1$, the coating has a constant dielectric permittivity $\varepsilon_{2}=2$ and thickness $b-a=10 \mathrm{~nm}$, and the core is metallic with: (a) a Ag core and (b) a Au core. The dipole moment of the emitter is radial. The insets of both panels show the different contributions of the radiative and SP modes to the total SE rate in the absence of losses, for $a=40 \mathrm{~nm}$ and $b=50 \mathrm{~nm}$.

losses are considered, there is an additional contribution from lossy surface modes, especially very close to the surface of the core.

In Fig. 6 we consider the effect of different values of the dielectric permittivity of the coating on the SE rate. The $x$ axis shows the radial position of the emitter. In Fig. 6(a) we consider a Ag core and in Fig. 6(b) a Au core, where the coated cylinder has a core radius $a=40 \mathrm{~nm}$ and an outer radius $b=50 \mathrm{~nm}$. In both panels the normalized SE rate, $\tilde{\gamma}$, increases as we increase the dielectric permittivity of the coating. The emission wavelength of the emitter is chosen to be close to the SP wavelength and as we increase the dielectric permittivity, the SP wavelength redshifts, as can be seen in the legends in Fig. 6. This redshift is due to the fact that the SP condition depends on $\varepsilon_{2}$ (for a planar interface, this condition is $\operatorname{Re}\left(\varepsilon_{1}\right)=-\varepsilon_{2}$ at $\left.\omega_{\mathrm{SP}}\right)$.

\section{B. Energy transfer function}

In this section we consider the ET function for a pair of quantum emitters where the donor is excited and the acceptor is in the ground state. The donor is at a fixed position, and the position of the acceptor is varied.

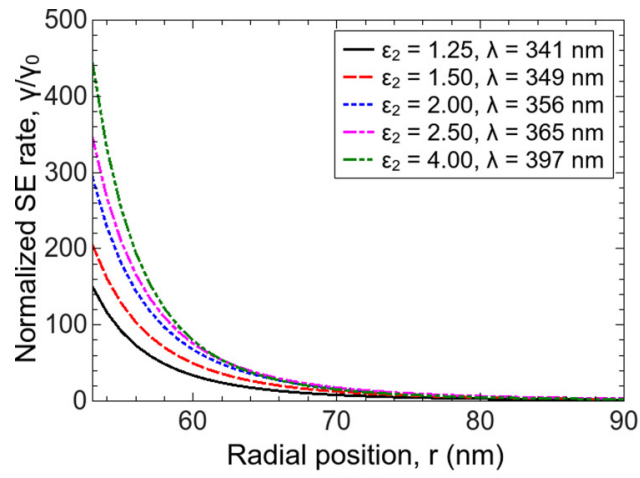

(a)
In Fig. 7 we present a contour plot of the normalized ET function for four different coated cylinder geometries. The figure shows an $x y$ cross section of a coated cylinder with a core radius $a=40 \mathrm{~nm}$ and an outer radius $b=50 \mathrm{~nm}$. The position of the donor is fixed on the $x$ axis at $r_{\mathrm{D}}=55 \mathrm{~nm}$ and is indicated by the arrow. The ET function is calculated for the acceptor positioned at each point in the plot. The emission wavelength of the donor has been chosen close to the SP wavelength in each case.

We first consider the $\mathrm{Ag}$ core and $\mathrm{Au}$ core geometries, shown in Figs. 7(a) and 7(b) respectively. The donor wavelength, $\lambda_{\mathrm{D}}$, is $356 \mathrm{~nm}$ for the Ag core and $520 \mathrm{~nm}$ for the $\mathrm{Au}$ core. Figure 7(a) shows that the normalized ET function, $\tilde{\Gamma}$, is concentrated in hot spots around the circumference of the coated cylinder. The number of hot spots is associated with the contributions of modes of different orders, $n$, of the Bessel and Hankel functions. In general, the number of hot spots, $\delta$, is given by $\delta=2 n+1$. It can be seen that excitation of SP modes can significantly enhance the ET function by up to 4 orders of magnitude.

Figure 7(b) shows the normalized ET function, $\tilde{\Gamma}$, when the material of the core is Au. The behavior is similar to the case of a Ag core, showing the same number of hot spots around

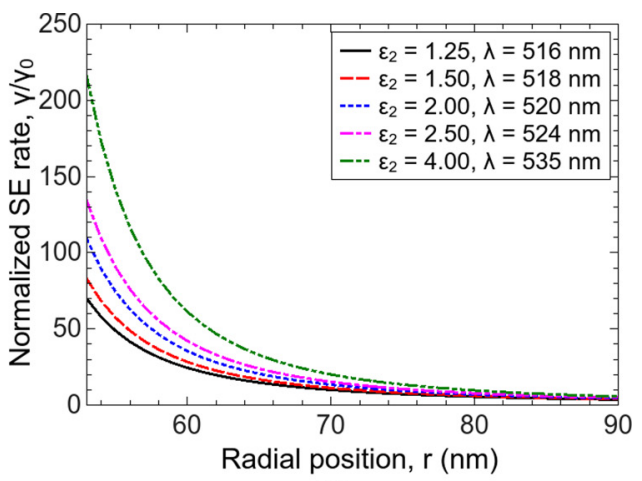

(b)

FIG. 6. (Color online) Normalized SE rate, $\tilde{\gamma}=\gamma / \gamma_{0}$, as a function of the radial position of the emitter, $r$, for several dielectric permittivities of the coating. The surrounding medium is air, $\varepsilon_{3}=1$, the coating thickness is $b-a=10 \mathrm{~nm}$, and the core is metallic, with (a) a Ag core and (b) a Au core. The radius of the core is $a=40 \mathrm{~nm}$. The dipole moment of the emitter is radial. 


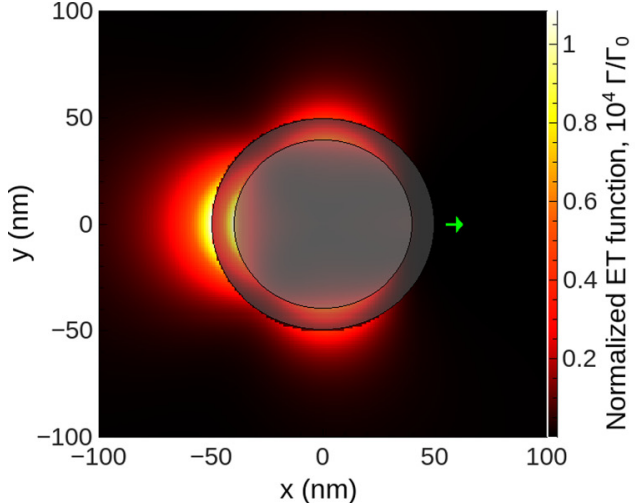

(a)

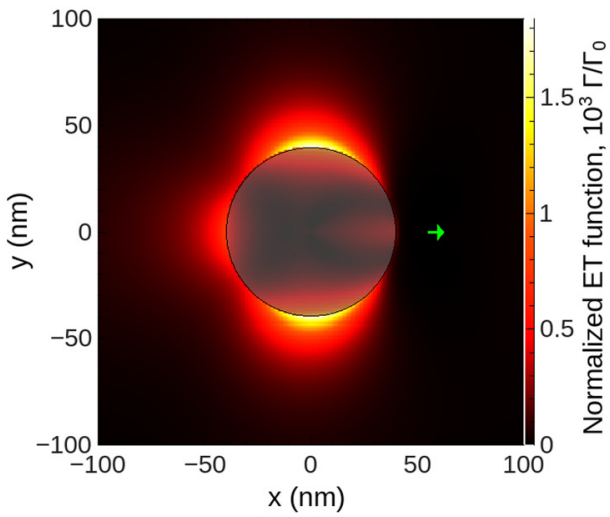

(c)

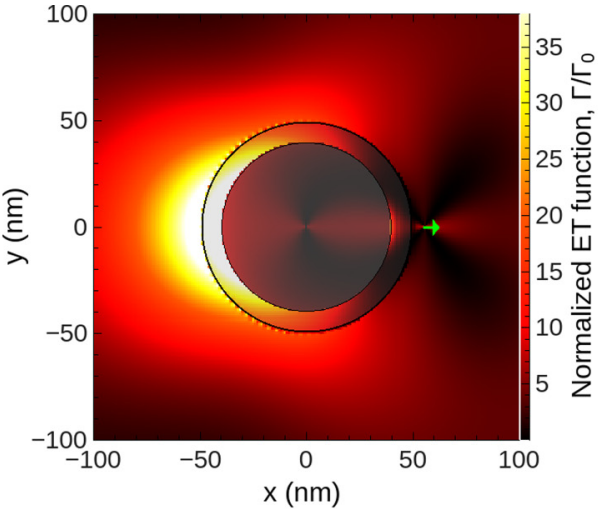

(b)

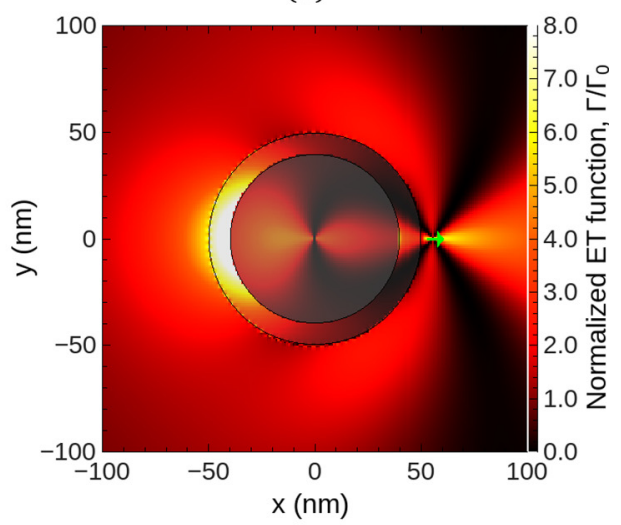

(d)

FIG. 7. (Color online) An $x y$ contour plot of the normalized ET function, $\tilde{\Gamma}$, near a coated metallic cylinder with a core radius $a=40 \mathrm{~nm}$ and an outer radius $b=50 \mathrm{~nm}$. The dielectric permittivity of the coating is $\varepsilon_{2}=2$ and the surrounding medium is air, $\varepsilon_{3}=1$. The position of the donor is fixed for all panels at $r_{\mathrm{D}}=55 \mathrm{~nm}$ on the $x$ axis. The material of the core cylinder and the emission wavelength of the donor are different for each panel: (a) Ag core with $\lambda_{\mathrm{D}}=356 \mathrm{~nm}$; (b) Au core with $\lambda_{\mathrm{D}}=520 \mathrm{~nm}$; (c) Ag core, no coating and $\lambda_{\mathrm{D}}=340 \mathrm{~nm}$; (d) dielectric core with $\varepsilon_{1}=4$ and $\lambda_{\mathrm{D}}=356 \mathrm{~nm}$. For each case where the core is metallic, the emission wavelength matches the SP wavelength. Both the donor and the acceptor dipole moments are radial.

the circumference. However, because of the higher losses in $\mathrm{Au}$, the enhancement across the cylinder is much lower.

To investigate the influence of the dielectric coating on the ET function we have considered two additional cases, that of a metallic core with no coating and that of a simple dielectric cylinder. Figure 7(c) shows a contour plot of the $x y$ cross section of the normalized ET function for a $\mathrm{Ag}$ cylinder of radius $a=40 \mathrm{~nm}$, for which the position of the donor is the same as in Fig. 7(a). The emission wavelength of the donor is chosen close to the SP wavelength for this case, which is $\lambda_{\mathrm{SP}}=340 \mathrm{~nm}$. It is clear that in the absence of the dielectric coating, the normalized ET function, $\tilde{\Gamma}$, across the Ag cylinder is considerably smaller. Therefore, for the case of the coated metal core cylinder the dipole near field is able to couple with the coating. Additionally, the dielectric material enhances the strength of the near field, as we have already shown in the section on the SE rate, i.e. Fig. 6.

It is also instructive to consider a purely dielectric cylinder, where we replace the metallic core with a material with a constant dielectric permittivity, $\varepsilon_{1}=4$. The emission wavelength, $\lambda_{\mathrm{D}}=356 \mathrm{~nm}$, and the donor position are identical to those in Fig. 7(a) to allow for direct comparison. It can be seen that the normalized ET function, $\tilde{\Gamma}$, is significantly smaller than in the other panels. Outside the cylinder it has a value $\tilde{\Gamma} \approx 7$ very close to the dielectric interface and, as the distance is increased, it reverts to the free-space value. The importance of the excitation of the SP modes for a large enhancement of the ET function is thus evident when we compare the dielectric core case with the metallic core cases considered in the rest of Fig. 7. The reader should note the different orders of magnitude for the color maps shown in Fig. 7.

In Fig. 8 we investigate the effect of changing the size of the coated cylinder on the ET mechanism. The normalized ET function, $\tilde{\Gamma}$, is presented in a $x y$ cross section of the coated cylinder. In both panels the core material is $\mathrm{Ag}$, with $\varepsilon_{2}=2$ and $\varepsilon_{3}=1$. For Fig. 8(a) we have $a=20 \mathrm{~nm}, b=30 \mathrm{~nm}$, the position of the donor $r_{\mathrm{D}}=35 \mathrm{~nm}$ on the $x$ axis, and the emission wavelength of the donor is close to the SP wavelength at $\lambda_{\mathrm{D}}=356 \mathrm{~nm}$. It can be seen that the normalized ET function, $\tilde{\Gamma}$, exhibits one hot spot at a position on the opposite side of the cylinder relative to the donor and is confined around the periphery of the coated cylinder. When we consider larger dimensions for the coated cylinder, $a=80 \mathrm{~nm}$, and $b=90 \mathrm{~nm}$ as shown in Fig. 8(b), the number of hot spots increases and the spatial extent of the enhancement of the normalized ET function, $\tilde{\Gamma}$, also increases. For Fig. 8(b), the position of the donor is $r_{\mathrm{D}}=100 \mathrm{~nm}$ on the $x$ axis and the donor emission wavelength is close to the SP wavelength, 


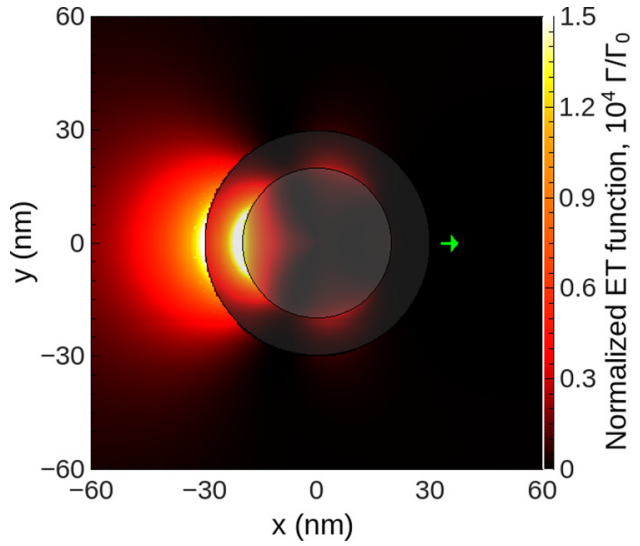

(a)

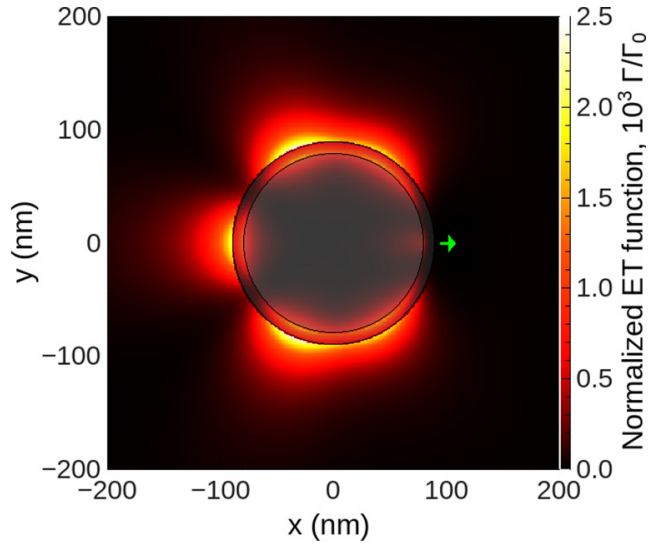

(b)

FIG. 8. (Color online) An $x y$ contour plot of the normalized ET function, $\tilde{\Gamma}$, near a coated cylinder with a Ag core, and a dielectric coating $\left(\varepsilon_{2}=2\right)$, embedded in air $\left(\varepsilon_{3}=1\right)$. (a) $a=20 \mathrm{~nm}, b=30 \mathrm{~nm}, r_{\mathrm{D}}=35 \mathrm{~nm}$; (b) $a=80 \mathrm{~nm}, b=90 \mathrm{~nm}, r_{\mathrm{D}}=100 \mathrm{~nm}$. Both the donor and the acceptor dipole moments are radial and the donor emission wavelength is $\lambda_{\mathrm{D}}=356 \mathrm{~nm}$.

$\lambda_{\mathrm{D}}=356 \mathrm{~nm}$. The number of hot spots is given by the coupling to SPs of different orders $n$, the dispersion curves of which are shown in Fig. 2.

\section{Energy transfer efficiency}

In the previous two sections we have considered generic donor-acceptor pairs with $\delta$-like emission and absorption spectra, i.e., single-frequency spectra. As such, the normalized SE or ET rates were determined by the optical response of the coated metallic cylinder, through the Green's tensor; see Eqs. (3) and (6).

We now relax this constraint and consider realistic donor emission spectra, $f_{\mathrm{D}}(\lambda)$, and acceptor absorption spectra, $\sigma_{\mathrm{A}}(\lambda)[53,54]$. These spectra describe statistical ensembles of donor-acceptor pairs.

When the donor dipole is excited it has two ways of relaxing to the ground state: It can either transfer its excitation energy to the acceptor dipole with an ET rate $k_{\mathrm{ET}}$ or it can relax with decay rate $k_{\mathrm{SE}}$. The decay rate $k_{\mathrm{SE}}$ takes account of photon emission into the far-field, intrinsic nonradiative recombination paths, coupling to SP modes and losses in the metallic core. The SE and ET processes are, therefore, in competition with each other and we introduce an ET efficiency to describe this competition. We will consider, in what follows, donors with a quantum yield of one, $Y_{0}=1.0$, which assumes no intrinsic losses such as phonon relaxation, etc.

Using the expressions we have introduced in Eqs. (4) and (7) for the SE and ET rates of ensembles of emitters and donoracceptor pairs, we now define an ET efficiency $\eta$ as [55]

$$
\eta=\frac{k_{\mathrm{ET}}}{k_{\mathrm{SE}}+k_{\mathrm{ET}}} .
$$

This quantity gives the relative contribution of the ET process to the total decay rate of the donor. If the ET efficiency, $\eta$, has a value larger than $\eta>50 \%$, then the decay of the excited state of the donor occurs mainly by ET to the acceptor, rather than relaxation into photon or SP modes.

We next consider two donor-acceptor pairs. The donor emission and acceptor absorption spectra are both given by a Gaussian distribution,

$$
A_{q i} e^{-\left(\lambda-\lambda_{q i}\right)^{2} / \Delta \lambda_{q i}^{2}},
$$

where $q=\mathrm{D}$ represents the donor, $q=\mathrm{A}$ represents the acceptor, $i=1$ corresponds to the on-resonance case, and $i=$ 2 corresponds to the off-resonance case. $A_{q i}$ is a normalization constant, $\lambda_{q i}$ gives the position of the spectral peak, and $\Delta \lambda_{q i}$ is the half width at half maximum (HWHM) of the spectrum. The normalization constant of the donor emission spectrum is given as $A_{\mathrm{D} i}^{-1}=\int_{0}^{\infty} d \lambda f_{\mathrm{D}}(\lambda)$. The HWHM will be $\Delta \lambda_{\mathrm{D} i}=$ $20 \mathrm{~nm}$ for both donor-acceptor pairs, which corresponds to a typical spectrum of a fluorescent dye, e.g., fluorescein [53]. The constant for the acceptor absorption spectrum is $A_{\mathrm{A} i}=$ $0.021 \mathrm{~nm}^{2}$, while the HWHM is $\Delta \lambda_{\mathrm{A} i}=50 \mathrm{~nm}$. The preceding values are common to both donor-acceptor pairs. The two donor-acceptor pairs do, however, differ with respect to the positions of their emission and absorption peaks. For the first pair, the donor emission peak is at $\lambda_{\mathrm{D} 1}=363 \mathrm{~nm}$, while the absorption maximum is at $\lambda_{\mathrm{A} 1}=373 \mathrm{~nm}$; for the second pair we have $\lambda_{\mathrm{D} 2}=453 \mathrm{~nm}$ and $\lambda_{\mathrm{A} 2}=463 \mathrm{~nm}$. Figures $9(\mathrm{a})$ and 9 (b) show the normalized emission spectrum of the donor, $f_{\mathrm{D}}(\lambda)$, and the absorption cross section spectrum of the acceptor, $\sigma_{\mathrm{A}}(\lambda)$, together with the ET function, $\Gamma(\lambda)$. Since the ET function, $\Gamma(\lambda)$, is given by the Green's tensor of the coated metallic cylinder, Eq. (6), it is the same for both donor-acceptor pairs, with a peak at $\lambda \approx 365 \mathrm{~nm}$, close to the $\mathrm{SP}$ wavelength. The peak is redshifted from the SP wavelength, $\lambda_{\mathrm{SP}}=356 \mathrm{~nm}$, as due to the finite distance of the donor and acceptor from the surface of the core, the pair can only couple to SPs with smaller wave vectors, which have a lower frequency and hence a longer wavelength. The permittivity of the coating is the same, $\varepsilon_{2}=2$, and the background medium is air, $\varepsilon_{3}=1$. The donor emission spectrum, $f_{\mathrm{D}}(\lambda)$, and acceptor absorption spectrum, $\sigma_{\mathrm{A}}(\lambda)$, of the first donor-acceptor pair considered both have a good overlap with $\Gamma(\lambda)$, i.e., this pair is on-resonance. The overlap is poor for the second donor-acceptor pair; i.e., it is off-resonance.

The Förster radius, $R_{0}$, is defined as the donor-acceptor separation at which $\eta$ is $50 \%$ [56]. The Förster radius is calculated to be 6.07 and $7.04 \mathrm{~nm}$ in free space for the first and 


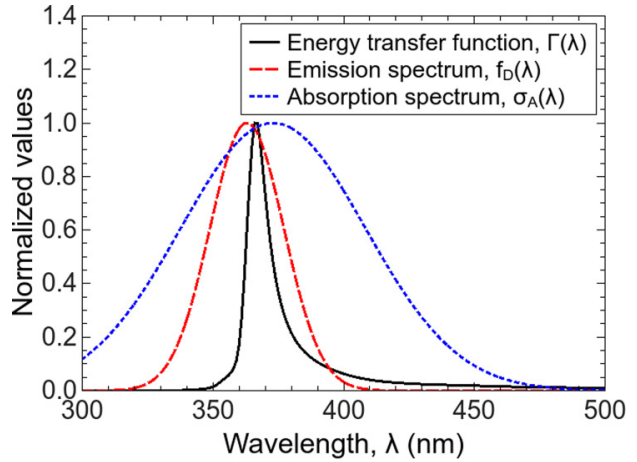

(a)



(c)

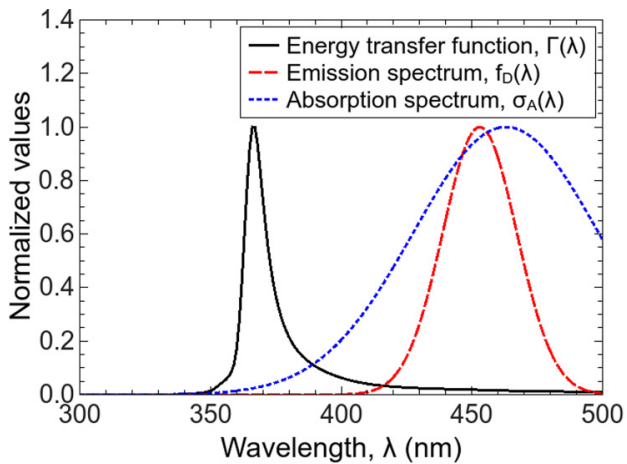

(b)

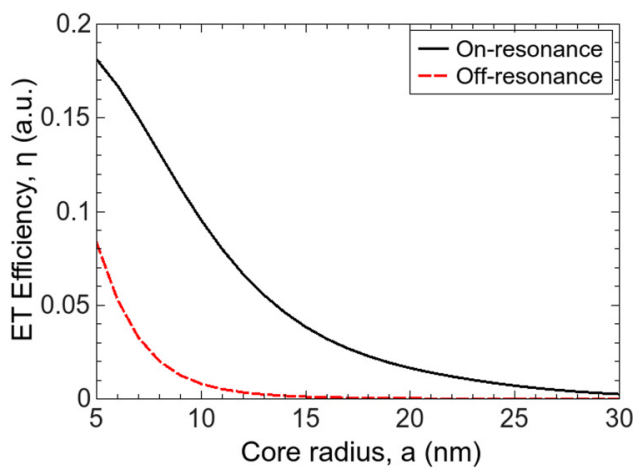

(d)

FIG. 9. (Color online) (a),(b) Normalized donor emission spectrum, $f_{\mathrm{D}}(\lambda)$, acceptor absorption spectrum, $\sigma_{\mathrm{A}}(\lambda)$, and ET function, $\Gamma(\lambda)$, for two different donor/acceptor pairs: (a) on-resonance and (b) off-resonance. (c) ET efficiency, $\eta$, for a coated metallic cylinder, with a core radius $a=10 \mathrm{~nm}$, outer radius $b=15 \mathrm{~nm}$, for a donor placed at $r_{\mathrm{D}}=20 \mathrm{~nm}$ on the $x$ axis, as a function of acceptor distance, $d$, from the core surface, diametrically opposite the donor. (d) ET efficiency, $\eta$, as a function of core radius, $a$, for an outer radius $b=a+5 \mathrm{~nm}$, donor position $r_{\mathrm{D}}=a+10 \mathrm{~nm}$ and acceptor position $r_{\mathrm{A}}=-a-2 \mathrm{~nm}$, both on the $x$ axis. The shaded region in (c) represents the coating.

second donor-acceptor pair, respectively. These values were calculated from the spectral overlap of the normalized donor emission and acceptor absorption spectra as

$$
R_{0}=\left[\frac{3 c}{32 \pi^{4} n^{4}} \int_{0}^{\infty} d \lambda \lambda^{2} f_{\mathrm{D}}(\lambda) \sigma_{\mathrm{A}}(\lambda)\right]^{1 / 6},
$$

where $n$ is the refractive index of the host medium, in our case air with $n=1$.

The next step will be to consider the influence of the coated cylinder on the ET efficiency, $\eta$. The core radius is $a=10 \mathrm{~nm}$ and the outer radius is $b=15 \mathrm{~nm}$. The position of the donor is kept fixed at $r_{\mathrm{D}}=20 \mathrm{~nm}$ on the $x$ axis. In Fig. 9(c) we plot the value of the ET efficiency, $\eta$, as a function of the radial position of the acceptor diametrically opposite to the donor, for the two donor-acceptor pairs. For the on-resonance case, the ET efficiency has a value of $\eta=23 \%$ close to the Ag-dielectric interface while for the off-resonance case [cf. Fig. 9(b)], the value of the ET efficiency is $\eta=1.3 \%$ close to the Ag-dielectric interface. These values can be compared with the free-space ET efficiency of $0.0077 \%$ and $0.017 \%$ at a donor-acceptor separation of $30 \mathrm{~nm}$, corresponding to the diameter of the coated metallic cylinder, for the first and second donor-acceptor pair, respectively. It can also be noted that the Förster radius in free-space for the first donor-acceptor pair is smaller than for the second. Therefore, the enhanced
ET range near the coated metallic cylinder is a consequence of the excitation of SP modes on the coated cylinder.

In Fig. 9(d) we investigate the influence of the core radius, $a$, of the coated cylinder on the ET efficiency, $\eta$, by keeping the thickness of the coating fixed at $5 \mathrm{~nm}$. The dielectric permittivities of the coated cylinder layers are the same as before. The position of the donor is $r_{\mathrm{D}}=a+10 \mathrm{~nm}$, in air, and the position of the acceptor is $r_{\mathrm{A}}=-a-2 \mathrm{~nm}$, inside the coating. In Fig. 9(d) we observe that for the case where the emission and absorption spectra are on resonance, the ET efficiency reaches values as high as $\eta=19.4 \%$ at $a=5 \mathrm{~nm}$ and it has values $\eta>10 \%$ for core radii $a<10 \mathrm{~nm}$. When the donor-acceptor pair from Fig. 9(b) is considered, we observe that the maximum values of the ET efficiency are smaller, $\eta=7.9 \%$ at $a=5 \mathrm{~nm}$, and it drops off more abruptly as a function of core size. Comparing the on- and off-resonance cases shows that a large overlap of the donor emission spectrum and acceptor absorption cross section with the SP spectrum of the ET function, $\Gamma(\lambda)$, can significantly enhance the ET efficiency, $\eta$, and mediate the interactions over larger distances.

In Fig. 10(a) we consider the influence of the dielectric permittivity of the coating, $\varepsilon_{2}$, on the SE rate of a donor, $k_{\mathrm{SE}}$, and the ET rate between a donor and an acceptor, $k_{\mathrm{ET}}$, for the geometry considered in the previous paragraph with parameters $a=10 \mathrm{~nm}$ and $b=15 \mathrm{~nm}$. For the donor emission and acceptor absorption spectra, we use the data from Fig. 9(a), the on-resonance case. The donor is located at $r_{\mathrm{D}}=20 \mathrm{~nm}$ and 


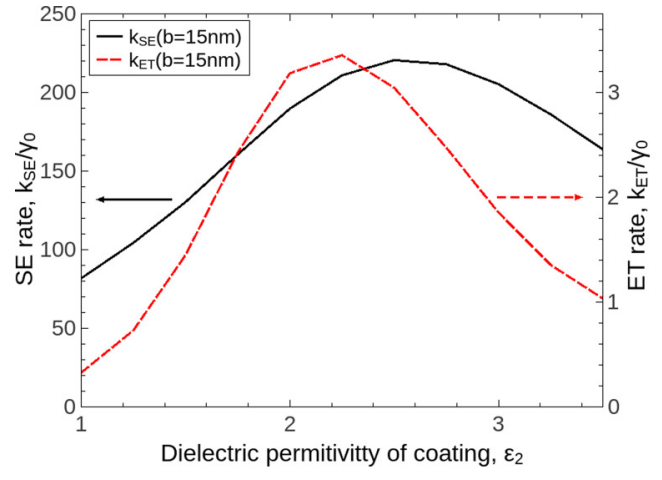

(a)

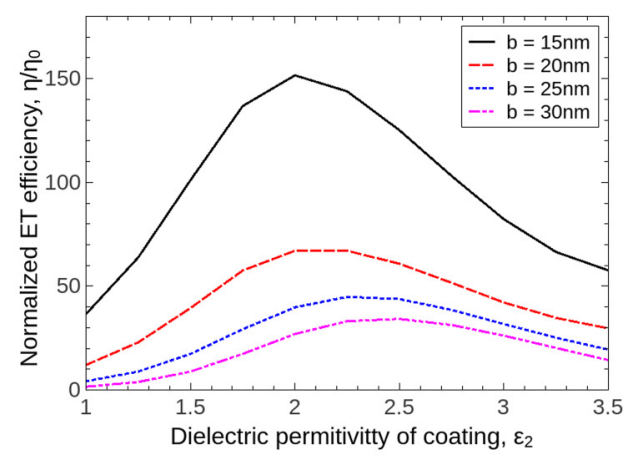

(b)

FIG. 10. (Color online) Coated cylinder of inner radius, $a$, donor position $r_{\mathrm{D}}=b+5 \mathrm{~nm}$ and acceptor position $r_{\mathrm{A}}=-b-5 \mathrm{~nm}$, both on the $x$ axis. (a) SE rate, $k_{\mathrm{SE}}$, of the donor and $\mathrm{ET}$ rate, $k_{\mathrm{ET}}$, between donor-acceptor for outer radius $b=15 \mathrm{~nm}$; (b) normalized ET efficiency, $\tilde{\eta}$, as a function of the dielectric permittivity of the coating, for several values of the coating thickness.

the acceptor is located at $r_{\mathrm{A}}=-20 \mathrm{~nm}$, both on the $x$ axis. The SE and ET rates are expressed in units of free-space $\mathrm{SE}, \gamma_{0}$. The $\mathrm{SE}$ rate, $k_{\mathrm{SE}}$, is two orders of magnitude larger compared to the ET rate, $k_{\mathrm{ET}}$. This is due to the fact that the distance between the donor and the acceptor is large and thus the factor $\left|\mathbf{n}_{\mathrm{A}} \cdot \mathfrak{G}\left(\mathbf{r}_{\mathrm{A}}, \mathbf{r}_{\mathrm{D}}, \omega\right) \cdot \mathbf{n}_{\mathrm{D}}\right|^{2}$ of Eq. (7) is weak compared with the factor $\gamma(\lambda)$ of Eq. (4). The SE rate reaches a maximum value at $\varepsilon_{2}=2.5$, while the maximum value of the ET rate, $k_{\mathrm{ET}}$, is attained at $\varepsilon_{2}=2.25$. The interplay between the SE and the ET contributions to the ET efficiency, $\eta$, will determine for which value of the dielectric permittivity of the coating, $\varepsilon_{2}$, the ET efficiency reaches its maximum, as seen in Fig. 10(b).

Figure 10(b) shows the influence of the dielectric permittivity of the coating, $\varepsilon_{2}$, and its thickness, on the ET efficiency, $\eta$, for the same inner core radius, $a$, and donor and acceptor spectral properties. The donor position is $r_{\mathrm{D}}=b+5 \mathrm{~nm}$ and the acceptor position $r_{\mathrm{A}}=-b-5 \mathrm{~nm}$, both on the $x$ axis. In order to quantify the influence of the coated cylinder geometry on the ET efficiency, we introduce the normalized ET efficiency, $\tilde{\eta}$, as $\tilde{\eta}=\eta / \eta_{0}$, where $\eta$ is the ET efficiency in the presence of the coated cylinder and $\eta_{0}$ is the free-space ET efficiency. The normalized ET efficiency, $\tilde{\eta}$, decreases as we increase the outer radius, $b$, due to the decoupling between the near field of the quantum emitters and the SP modes of the metallic core. As the outer radius, $b$, is increased even more, the ET efficiency reverts to its free-space value and we have $\tilde{\eta} \rightarrow 1$. The peak in the normalized ET efficiency for $b=15 \mathrm{~nm}$ occurs at a value of the dielectric permittivity of the coating of $\varepsilon_{2}=2$. By varying the dielectric permittivity, the ET function is shifted in wavelength. The $\varepsilon_{2}=2$ is the value for the on-resonance case, as shown in Fig. 9(a). Furthermore, as we increase the outer radius, $b$, we see that the peak in the normalized ET efficiency is shifted from $\varepsilon_{2}=2$ to $\varepsilon_{2}=2.5$, due to the redshifting of the SP resonance wavelength with increasing outer radius $b$. Thus, the dielectric permittivity of the coating, $\varepsilon_{2}$, and the thickness of the coating, constitute additional parameters that can be adjusted to optimally couple donor-acceptor pairs with different optical properties, maximizing the ET efficiency.

\section{SUMMARY AND CONCLUSIONS}

In this paper we have presented the role of a dielectric coated metallic cylinder in modifying the SE rate of a single emitter and the ET rate in a donor-acceptor pair. The excitation of SP modes, when the emission wavelength is close to the SP wavelength, enhances the SE and ET rates and functions by several orders of magnitude compared with their free-space values.

First, we considered the SE rate of an emitter in the presence of a coated cylinder. We saw that the SE rate is enhanced through the excitation of SP modes. The enhancement is larger when the emitter is in close proximity to the metallic core and its emission wavelength is close to the SP wavelength. The SE rate also depends on the radius of the core, and is seen to increase with the radius when the emission wavelength is close to the SP wavelength. When the dielectric permittivity of the coating is increased, the SP wavelength is redshifted, as expected. The maximum of the SE rate follows the same trend, undergoing a redshift. Furthermore, the value of this maximum increases with the dielectric permittivity of the coating.

We next considered the ET function between a donoracceptor pair. By exciting SP modes, the ET function can be enhanced by orders of magnitude, the enhancement being localized at hot spots around the circumference of the coated cylinder. The number of these hot spots is in direct relation to the order of the SP mode or modes being excited. The presence of the coating dielectric layer around the metallic core further increases the ET enhancement provided by the core by itself.

Furthermore, since SE and ET are competitive processes, we considered the ET efficiency, $\eta$, to investigate the interplay between them. As examples, we chose two donor-acceptor pairs, with different donor emission and acceptor absorption spectra. For the first pair, the emission and absorption spectra overlap with the ET function, given by the Green's tensor, while for the second pair, they do not. By considering a fixed geometry for the coated cylinder and varying the position of the acceptor and the core radius, $a$, a significant difference between on- and off-resonance emission spectrum for SP mode excitation is evident.

Additionally, we have shown that the SP-mediated coupling between different donor-acceptor pairs can be engineered 
in a predictable way by controlling the refractive index of the coating, as well as its thickness. These parameters can be chosen such that one can obtain an optimal coupling between donors and acceptors with specific optical properties.

For applications in which the ET efficiency needs to be optimized, we have shown that fine tuning of the geometrical parameters of the dielectric coated metallic cylinder, e.g., thickness of dielectric coating, can lead to large enhancements of the ET rate. Control of the wavelength at which the ET efficiency is maximum can be achieved through the refractive index of the dielectric coating and, for a more coarse-grained tuning, through the material of the core, i.e., Ag vs Au.

\section{ACKNOWLEDGMENT}

This work was supported by the Science Foundation Ireland under Grant No. 10/IN.1/12975.

\section{APPENDIX A: CHARACTERISTIC MATRIX AND VECTORS}

In this appendix we give the expressions for the characteristic matrix and the free-term vectors from Eq. (11). These expressions have been given in various articles (cf. [52,57]). The characteristic matrix, $\Delta\left(n, k_{z}\right)$, has the form

$$
\Delta=\left(\begin{array}{cccccccc}
k_{\rho 1} J_{n 1 a}^{\prime} & \frac{n k_{z}}{a k_{1}} J_{n 1 a} & -k_{\rho 2} H_{n 2 a}^{\prime(1)} & -\frac{n k_{z}}{a k_{2}} H_{n 2 a}^{(1)} & -k_{\rho 2} J_{n 2 a}^{\prime} & -\frac{n k_{z}}{a k_{2}} J_{n 2 a} & 0 & 0 \\
0 & \frac{k_{\rho 1}^{2}}{k_{1}} J_{n 1 a} & 0 & -\frac{k_{\rho 2}}{k_{2}} H_{n 2 a}^{(1)} & 0 & -\frac{k_{\rho 2}^{2}}{k_{2}} J_{n 2 a} & 0 & 0 \\
\frac{n k_{z}}{a} J_{n 1 a} & k_{1} k_{\rho 1} J_{n 1 a}^{\prime} & -\frac{n k_{z}}{a} H_{n 2 a}^{(1)} & -k_{2} k_{\rho 2} H_{n 2 a}^{\prime(1)} & -\frac{n k_{z}}{a} J_{n 2 a} & k_{2} k_{\rho 2} J_{n 2 a}^{\prime} & 0 & 0 \\
k_{\rho 1}^{2} J_{n 1 a} & 0 & -k_{\rho 2}^{2} H_{n 2 a}^{(1)} & 0 & -k_{\rho 2}^{2} J_{n 2 a} & 0 & 0 & 0 \\
0 & 0 & 0 & \frac{k_{\rho 2}^{2}}{k_{2}} H_{n 2 b}^{(1)} & 0 & \frac{k_{\rho 2}^{2}}{k_{2}} J_{n 2 b} & 0 & -\frac{k_{\rho 3}^{2}}{k_{3}} H_{n 3 b}^{(1)} \\
0 & 0 & k_{\rho 2} H_{n 2 b}^{\prime(1)} & \frac{n k_{z}}{b k} H_{n 2 b}^{(1)} & k_{\rho 2} J_{n 2 b}^{\prime} & \frac{n k_{z}}{a k_{2}} J_{n 2 b} & -k_{\rho 3} H_{n 3 b}^{\prime(1)} & -\frac{n k_{z}}{k_{3} b} H_{n 3 b}^{(1)} \\
0 & 0 & k_{\rho 2}^{2} H_{n 2 b}^{(1)} & 0 & k_{\rho 2}^{2} J_{n 2 b} & 0 & -k_{\rho 3}^{2} H_{n 3 b}^{(1)} & 0 \\
0 & 0 & \frac{n k_{z}}{b} H_{n 2 b}^{(1)} & k_{2} k_{\rho 2} H_{n 2 b}^{\prime(1)} & -\frac{n k_{z}}{b} J_{n 2 b} & k_{2} k_{\rho 2} J_{n 2 b}^{\prime} & -\frac{n k_{z}}{b} H_{n 3 b}^{(1)} & -k_{3} k_{\rho 3} H_{n 3 b}^{\prime(1)}
\end{array}\right),
$$

where we use the compact notation $J_{n 1 a}=J_{n}\left(k_{\rho 1} a\right), H_{n 2 a}^{(1)}=H_{n}^{(1)}\left(k_{\rho 2} a\right)$, and so on. The free-term vectors, $\mathbf{V}_{M}^{3}\left(n, k_{z}\right)$ and $\mathbf{V}_{N}^{3}\left(n, k_{z}\right)$, have the form

$$
\mathbf{V}_{M}^{3}\left(n, k_{z}\right)=\left(\begin{array}{c}
0 \\
0 \\
0 \\
0 \\
0 \\
k_{\rho 3} J_{n}^{\prime}\left(k_{\rho 3} b\right) \\
k_{\rho 3}^{2} J_{n}\left(k_{\rho 3} b\right) \\
\frac{n k_{z}}{b} J_{n}\left(k_{\rho 3} b\right)
\end{array}\right), \quad \mathbf{V}_{N}^{3}\left(n, k_{z}\right)=\left(\begin{array}{c}
0 \\
0 \\
0 \\
0 \\
\frac{k_{\rho 3}^{2}}{k_{3}} J_{n}\left(k_{\rho 3} b\right) \\
\frac{n k_{z}}{b k_{3}} J_{n}\left(k_{\rho 3} b\right) \\
0 \\
k_{3} k_{\rho 3} J_{n}^{\prime}\left(k_{\rho 3} b\right)
\end{array}\right) \text {, }
$$

while the coefficients vectors are

$$
\mathbf{R}_{M}=\left(\begin{array}{c}
R_{M M}^{13} \\
R_{N M}^{13} \\
R_{M M}^{H 23} \\
R_{N M}^{H 23} \\
R_{M M}^{J 23} \\
R_{N M}^{H 23} \\
R_{M M}^{33} \\
R_{M M}^{33}
\end{array}\right), \quad \mathbf{R}_{N}=\left(\begin{array}{c}
R_{M N}^{13} \\
R_{N N}^{13} \\
R_{M N}^{H 23} \\
R_{N N}^{H 23} \\
R_{M N}^{J 23} \\
R_{N N}^{H 23} \\
R_{M N}^{33} \\
R_{M N}^{33}
\end{array}\right)
$$

Using these expressions, Eq. (11) can be readily solved.

\section{APPENDIX B: SP CONTRIBUTION TO THE DECAY RATES}

In this appendix we present the formalism used to extract the SP contribution to the normalized SE rate, $\tilde{\gamma}$, from Eq. (3). These contributions are shown in the insets of the panels in Fig. 5. To extract the SP contribution, we make use of the dispersion diagram in Fig. 2(a) and the relevant geometrical and material parameters. For a specific emission frequency of the quantum emitter the dispersion diagram determines the number of SP modes that can be excited by the emitter and their wave number. As Fig. 2(a) shows, the quantum system cannot excite any SP mode when its emission frequency is above the SP frequency $\omega_{\mathrm{SP}}=5.3 \mathrm{fs}^{-1}$. For emission frequencies smaller than $\omega_{\min }=3.5 \mathrm{fs}^{-1}$, the quantum system can only couple to the fundamental mode, $n=0[17,20]$. 
We consider the case where we ignore the losses in the metallic core, to better show the SP contribution. By choosing the emission frequency of the quantum system in the interval $\left(\omega_{\min }, \omega_{\mathrm{SP}}\right)$, the SP with angular momentum numbers $n=0,1$ can be excited. These SPs have wave numbers $k_{\mathrm{SP} 0}$ and $k_{\mathrm{SP} 1}$, respectively, which correspond to two poles of the generalized scattering coefficients from Eq. (A3). The integrand from Eq. (12) when $\mathbf{r}=\mathbf{r}_{s}=r$ has the general expression

$$
d \mathfrak{G}_{n}^{i i}\left(k_{z}, r\right)=\frac{1}{\operatorname{Det}\left[\Delta_{n}\left(k_{z}\right)\right]} F_{n}^{i i}\left(k_{z}, r\right),
$$

where $i=1,2,3$ for the three different media and $F_{n}^{i i}\left(k_{z}, r\right)$ is a function containing all the different contributions to the integrand, except the pole contribution, which is given by the determinant of the characteristic matrix. Using Cauchy's residue theorem, the contributions of the two poles to the Green's tensor can be written as

$$
\begin{aligned}
& \mathfrak{G}_{s(r r)}^{i i}(\mathbf{r}, \mathbf{r}, \omega)_{\mathrm{SP}} \\
& =\pi i\left(\frac{F_{0}^{i i}\left(k_{\mathrm{SP} 0}, r\right)}{\left.\frac{\partial}{\partial k_{z}}\left\{\operatorname{Det}\left[\Delta_{0}\left(k_{z}\right)\right]\right\}\right|_{k_{z}=k_{\mathrm{SP} 0}}}+\frac{2 F_{1}^{i i}\left(k_{\mathrm{SP} 0}, r\right)}{\left.\frac{\partial}{\partial k_{z}}\left\{\operatorname{Det}\left[\Delta_{1}\left(k_{z}\right)\right]\right\}\right|_{k_{z}=k_{\mathrm{SP} 1}}}\right),
\end{aligned}
$$

where the factor of 2 for the second SP contribution comes from the fact that the $n=1$ and $n=-1$ modes have equal contributions.
[1] E. Purcell, Phys. Rev. 69, 681 (1946).

[2] D. T. Alves, C. Farina, and A. C. Tort, Phys. Rev. A 61, 034102 (2000).

[3] R. Matloob, Phys. Rev. A 62, 022113 (2000).

[4] R. M. Amos and W. L. Barnes, Phys. Rev. B 55, 7249 (1997).

[5] T. Kobayashi, Q. Zheng, and T. Sekiguchi, Phys. Lett. A 199, 21 (1995).

[6] C. A. Marocico and J. Knoester, Phys. Rev. A 84, 053824 (2011).

[7] J. M. Wylie and J. E. Sipe, Phys. Rev. A 30, 1185 (1984).

[8] M. Cho and R. J. Silbey, Chem. Phys. Lett. 242, 291 (1995).

[9] R. Carminati, J.-J. Greffet, C. Henkel, and J. Vigoureux, Opt. Commun. 261, 368 (2006).

[10] H. Mertens, A. F. Koenderink, and A. Polman, Phys. Rev. B 76, 115123 (2007).

[11] A. O. Govorov, J. Lee, and N. A. Kotov, Phys. Rev. B 76, 125308 (2007).

[12] P. Anger, P. Bharadwaj, and L. Novotny, Phys. Rev. Lett. 96, 113002 (2006).

[13] H. T. Dung, L. Knöll, and D.-G. Welsch, Phys. Rev. A 62, 053804 (2000).

[14] W. Żakowicz and M. Janowicz, Phys. Rev. A 62, 013820 (2000).

[15] V. Bordo, J. Opt. Soc. Am. B 29, 1799 (2012).

[16] D. P. Fussell, R. C. McPhedran, and C. Martijn de Sterke, Phys. Rev. A 71, 013815 (2005).

[17] D. E. Chang, A. S. Sørensen, P. R. Hemmer, and M. D. Lukin, Phys. Rev. B 76, 035420 (2007).

[18] U. Schröter and A. Dereux, Phys. Rev. B 64, 125420 (2001).

[19] C. A. Marocico and J. Knoester, Phys. Rev. A 79, 053816 (2009).

[20] D. Dzsotjan, A. S. Sørensen, and M. Fleischhauer, Phys. Rev. B 82, 075427 (2010).

[21] L. Novotny and C. Hafner, Phys. Rev. E 50, 4094 (1994).

[22] J. Barthes, A. Bouhelier, A. Dereux, and G. C. D. Francs, Sci. Rep. 3, 2734 (2013).

[23] K. H. Drexhage, J. Lumin. 1-2, 693 (1970).

[24] R. R. Chance, A. H. Miller, A. Prock, and R. Silbey, J. Chem. Phys. 63, 1589 (1975).

[25] J. Gersten and A. Nitzan, J. Chem. Phys. 75, 1139 (1981).

[26] J. I. Gersten and A. Nitzan, Chem. Phys. Lett. 104, 31 (1984).

[27] L. M. Folan, S. Arnold, and S. D. Druger, Chem. Phys. Lett. 118, 322 (1985).
[28] S. D. Druger, S. Arnold, and L. M. Folan, J. Chem. Phys. 87, 2649 (1987).

[29] M. V. Erementchouk, L. I. Deych, H. Noh, H. Cao, and A. A. Lisyansky, J. Phys.: Condens. Matter 21, 175401 (2009).

[30] G. S. Agarwal and S. Dutta Gupta, Phys. Rev. A 57, 667 (1998).

[31] P. Andrew and W. L. Barnes, Science 290, 785 (2000).

[32] D. M. Basko, F. Bassani, G. C. La Rocca, and V. M. Agranovich, Phys. Rev. B 62, 15962 (2000).

[33] D. Basko, G. C. La Rocca, F. Bassani, and V. Agranovich, Phys. Status Solidi 190, 379 (2002).

[34] W. L. Barnes, A. Dereux, and T. W. Ebbesen, Nature (London) 424, 824 (2003).

[35] S. V. Gaponenko, Introduction to Nanophotonics (Cambridge University Press, New York, 2010).

[36] C. T. Tai, Dyadic Green Functions in Electromagnetic Theory (IEEE Press, New York, 1994).

[37] W. C. Chew, Waves and Fields in Inhomogeneous Media (IEEE Press, New York, 1995).

[38] P. B. Johnson and R. W. Christy, Phys. Rev. B 6, 4370 (1972).

[39] M. Law, L. E. Greene, J. C. Johnson, R. Saykally, and P. Yang, Nat. Mater. 4, 455 (2005).

[40] O. L. Muskens, J. G. Rivas, R. E. Algra, E. P. A. M. Bakkers, and A. Lagendijk, Nano Lett. 8, 2638 (2008).

[41] C. B. Winkelmann, I. Ionica, X. Chevalier, G. Royal, C. Bucher, and V. Bouchiat, Nano Lett. 7, 1454 (2007).

[42] B. Zhang, H. Wang, L. Lu, K. Ai, G. Zhang, and X. Cheng, Adv. Funct. Mater. 18, 2348 (2008).

[43] F. De Angelis, M. Malerba, M. Patrini, E. Miele, G. Das, A. Toma, R. P. Zaccaria, and E. Di Fabrizio, Nano Lett. 13, 3553 (2013).

[44] Y. Estrin, D. H. Rich, A. V. Kretinin, and H. Shtrikman, Nano Lett. 13, 1602 (2013).

[45] M. Kadic, S. Guenneau, S. Enoch, and S. A. Ramakrishna, ACS Nano 5, 6819 (2011).

[46] Y. Ni, L. Gao, and C.-W. Qiu, Plasmonics 5, 251 (2010).

[47] D. E. Chang, A. S. Sørensen, E. A. Demler, and M. D. Lukin, Nat. Phys. 3, 807 (2007).

[48] H. T. Dung, L. Knöll, and D.-G. Welsch, Phys. Rev. A 65, 043813 (2002).

[49] H. T. Dung, L. Knöll, and D.-G. Welsch, Phys. Rev. A 57, 3931 (1998). 
[50] D. P. Craig and T. Thirunamachandran, Molecular Quantum Electrodynamics: An Introduction to Radiation Molecule Interactions (Academic Press, New York, 1998).

[51] X. Zhang, C. A. Marocico, M. Lunz, V. A. Gerard, Y. K. Gun'ko, V. Lesnyak, N. Gaponik, A. S. Susha, A. L. Rogach, and A. L. Bradley, ACS Nano 8, 1273 (2014).

[52] L.-W. Li, M.-S. Leong, T.-S. Yeo, and P.-S. Kooi, J. Electromagnet. Wave. 14, 961 (2000).
[53] L. Novotny and B. Hecht, Principles of Nano-optics, 2nd ed. (Cambridge University Press, Cambridge, UK, 2012).

[54] P. Andrew and W. L. Barnes, Science 306, 1002 (2004).

[55] X. Zhang, C. A. Marocico, M. Lunz, V. A. Gerard, Y. K. Gun'ko, V. Lesnyak, N. Gaponik, A. S. Susha, A. L. Rogach, and A. L. Bradley, ACS Nano 6, 9283 (2012).

[56] T. Förster, Ann. Phys. 437, 55 (1948).

[57] P. K. Rekdal, S. Scheel, P. L. Knight, and E. A. Hinds, Phys. Rev. A 70, 013811 (2004). 